\title{
Effect of memory and dynamical chaos in long Josephson junctions
}

\author{
K.N. Yugay, N.V. Blinov, and I.V. Shirokov \\ Omsk State University, Institute of Sensor Microelectronics \\ of Russian Academy of Sciences, Omsk 644077, Russia
}

\begin{abstract}
A long Josephson junction in a constant external magnetic field and in the presence of a dc bias current is investigated. It is shown that the system, simulated by the sine-Gorgon equation, "remembers" a rapidly damping initial perturbation and final asymptotic states are determined exactly with this perturbation. Numerical solving of the boundary sine-Gordon problem and calculations of Lyapunov indices show that this system has a memory even when it is in a state of dynamical chaos, i.e., dynamical chaos does not destroy initial information having a character of rapidly damping perturbation.

PACS number(s): 74.50+r, 05.45.+b
\end{abstract}

Dynamical chaos is one of the most interesting phenomena in the theory of Josephson junctions. ${ }^{1-10}$ This phenomenon is not only of theoretical importance but also of practical importance, because many devices are founded on Josephson junctions, in particular, superconducting quantum interference devices (SQUID's). ${ }^{11}$ Dynamical chaos in these devices is another source of noise. Furthemore, a long Josephson junction (LJJ) serves as a very good system for studying nonlinear phenomena such as an exitation of fluxons and antifluxons, their propagation, interaction, scattering, and breakup. Investigations of the last few years showed that a LJJ detects deeper characteristics that had seemed. Even in the simplest case, when a bias current and an external oscillating field are absent, the presence only of a constant external magnetic field leads to the most interesting phenomenon connected with the selection of the solution of the stationary Ferrell-Prange equation. The fact is that this equation has not only provided one solution by given boundary conditions; the number of these solutions increases by the strength of the external magnetic field and the total length of the junction. ${ }^{11}$ 
Recently we have shown ${ }^{12}$ that the selection of a solution is carried out with the form of a small and rapidly damping initial perturbation in time in the nonstationary sine-Gordon equation and what is more surprising an asymptotic solution of this equation coincides with one of the stable solutions of the stationary Ferrell-Prange equation. Two circumstances are remarkable here: (1) A small perturbation influences very much the evolution of the system with $t \rightarrow \infty$; in a sense it defines the character of asymtotic solutions. (2) One can say that in spite of the fact that a small perturbation is a rapidly damping one, the stable asymptotic solution "remembers" the initial perturbation. In other words, the nonlinear system, i.e., a LJJ, described with the sine-Gordon equation shows an effect of memory. However, in Ref. 12, the LJJ is studied solely under the influence of an external constant magnetic field. Therefore, it is of interest to investigate the LJJ from the point of view of the effect of memory not only in the presence of an external constant magnetic field but also under the influence of a dc bias current through the junction causing an exitation of dynamical chaos. How will the effect of memory in the presence of a dc bias current be shown? Will this effect take place in the states of dynamical chaos in general? Below we will try to give answers on these questions.

We write down the sine-Gordon equation in the presence of a dc bias current in a LJJ in the form

$$
\varphi_{t t}(x, t)+2 \gamma \varphi_{t}(x, t)-\varphi_{x x}(x, t)=-\sin \varphi(x, t)+\beta,
$$

where $\varphi(x, t)$ is the Josephson phase variable, $x$ is the distance along the junction normalized to the Josephson penetration length $\lambda_{J}$,

$$
\lambda_{J}=\left(\frac{c \Phi_{0}}{8 \pi^{2} j_{c} d}\right)^{1 / 2}
$$

$\Phi_{0}$ is the flux quantum, $j_{c}$ is the critical current density of the Josephson junction, $d=2 \lambda_{L}+b, \lambda_{L}$ is the London penetration length, $b$ is the thickness of the dielectric barrier, $t$ is the time normalized to the inverse of the Josephson plasma frequency $\omega_{J}$,

$$
\omega_{J}=\left(\frac{2 \pi c j_{c}}{C \Phi_{0}}\right)^{1 / 2}
$$

$C$ is the junction capacitance per unit area, $\gamma$ is the dissipative coefficient per unit area, and $\beta$ is the dc bias current density normalized to $j_{c}$. 
We write down the boundary condition for Eq. (1) in the form

$$
\begin{aligned}
\left.\frac{\partial \varphi(x, t)}{\partial x}\right|_{x=0} & \equiv H(0, t)=\left.\frac{\partial \varphi(x, t)}{\partial x}\right|_{x=L} \equiv H(L, t) \\
& =H_{0}\left(1-a e^{-t / t_{0}} \cos t\right),
\end{aligned}
$$

where $L$ is the total length of the junction normalized to $\lambda_{J}, H_{0}$ is the external constant magnetic field perpendicular to the junction and normalized as well as $H(0, t)$ and $H(L, t)$ to $\frac{\Phi_{0}}{2 \pi \lambda_{J} d}, a$ is the controlling (perturbation) parameter characterizing the rapidly damping in the time perturbation, and $t_{0}$ is the characteristic time of this damping perturbation normalized to $\omega_{J}^{-1}$.

Eq. (1) with boundary condition (2) is solved numerically. In contrast to the case $\beta=0$ considered by us ${ }^{12}$, the picture of magnetic field evolution in the junction turns out more complicated by $\beta \neq 0$ as will be shown below. Physically this is connected with the fact that the energy balance in the junction in the presence of bias current is such that the energy brought into the system with this current can make up for the energy loss because of dissipation or exceed it. And so one can expect that at few values of $\beta$ a state of junction will differ little from the stationary one described by the Ferrell-Prange equation. At sufficiently large values of $\beta$ asymptotic regular (periodic) solutions that represent waves - fluxons and antifluxons - moving along the junction and interacting among themselves and with junction boundaries and also nonregular solutions that represent dynamical chaos will take place ${ }^{1}$. Our calculations showed that if an asymtotic state at $a=0$ (further we shall call the state at $a=0$ as "starting") is regular, by introduction at the initial moment of a rapidly damping perturbation defined with the controlling parameter $a$ as well as in a stationary case, examined in Ref. 12, the selection of the asymtotic solution by a given set of parameters $H_{0}, \gamma, L$, and $\beta$ is determined with this parameter $a$; i.e., the system "remembers" the form of the rapidly damping perturbation and chooses the way of further evolution in accordance with this. (It is noteworthy that the effect of memory discussed here happens in a dissipative system and so it is not connected with the reproduction of a signal as it takes place, for example, in noncollision plasma in the effect of plasma echo ${ }^{14}$ ). Furthermore, our calculations show that if the "starting" state is regular (periodic), stationary states can also arise by introduction of perturbation $(a \neq 0)$ which it is of surprise in itself. However, the most remarkable fact is 
that the system is very sensitive to a rapidly damping perturbation as well as in the dynamical chaos conditions; i.e., the system has memory in this case too.

For the quantitative description of different characteristic states we used Lyapunov indices. We write down Eq. (1) in the form

$$
\left\{\begin{array}{l}
\varphi_{t}=V \\
V_{t}=-2 \gamma V+\varphi_{x x}-\sin \varphi+\beta
\end{array}\right.
$$

or, that is the same, in the form

$$
z_{t}=F(z)
$$

where $z$ is the vector with the components $\varphi$ and $V, z \equiv\left(\begin{array}{c}\varphi \\ V\end{array}\right)$, and $F(z)$ is defined as follows:

$$
F(z) \equiv F(\varphi, V)=\left(\begin{array}{l}
V \\
-2 \gamma+\varphi_{x x}-\sin \varphi+\beta
\end{array}\right) .
$$

Let $z(t)$ be a solution of Eq. (4). Then we can write down the equation for variations:

$$
\begin{aligned}
w_{t} & =\frac{\partial F(z(t))}{\partial z} w \\
w & \equiv\left(\begin{array}{l}
w_{1} \\
w_{2}
\end{array}\right)
\end{aligned}
$$

We define the Lyapunov index (LI) as

$$
\lambda=\lim _{t \rightarrow \infty} \frac{1}{t} \ln \frac{\|w(t)\|}{\|w(0)\|},
$$

where $\|w\|$ is the vector norm that we define as Euclidean norm

$$
\|w\|^{2}=\int_{0}^{L}\left(w_{1}^{2}+w_{2}^{2}\right) d x
$$

Depending on direction of the initial vector $w(0)$, different LI's will exist and their number will be infinite. The definition of LI (6) for system (3) is a natural generalization of a LI for finite-dimensional dynamic systems. ${ }^{13}$

The maximum LI plays a very important role because it is precisely this maximum that determines the motion character - exponential 
growth, decay, or zero change - for the majority of the trajectories of the system. A set of initial data $w(0)$ for which formula (6) gives LI's differing from the maximum one is negligibly small and by numerical calculations this formula gives the maximum LI as a rule.

The results a numerical solution of Eq. (1) with boundary conditions (2) and calculation of LI (6) showed that there exist three forms of characteristic states of the system for which the maximum LI can be as follows: (1) $\lambda>0,(2) \lambda<0$, and (3) $\lambda \leq 0$. The states with $\lambda>0$ represent the dynamical chaos states (Fig. 1), the states with $\lambda<0$ represent the stable stationary states (Fig.2), and the states with $\lambda \leq 0$ represent the regular (periodic) states (Fig. 3). All states in Figs. 1-3 that are shown as illustration are as "starting" ones $(a=0)$ and they are calculated with identical values of the parameters $H_{0}=1.25, L=5$, and $\gamma=0.26$, but with different values of $\beta$. For the chaos state in Fig. $1, \beta=0.50$; for the stationary one in Fig. $2, \beta=0.427$; and for the regular one in Fig. $3, \beta=0.60$. In Figs. $1-3$ the dependences of the potential $\varphi_{t}$ (potentials are normalized to the value $V_{c} \equiv \frac{\hbar \omega_{p}}{2 e}$ ) on time and the calculated values LI $\lambda$ corresponding to them are shown. We note that the "starting" chaotic state, represented in Fig. 1, is the same as in Ref. 1.

Let us examine the specific set of parameters, i.e., the definite point in parameter space, corresponding to the chaotic "starting" state: $H_{0}=$ $1.25, L=5, \gamma=0.26, \beta=0.44$, and $a=0$. If we input now the rapidly damping in the time perturbation determined by the controlling parameter $a$, the system does not remain in the previous chaotic state as the calculations show, but wanders between all three forms of states: chaotic, stationary, and regular, when this parameter changes. By calculations the following hierarchy of times was used: $t_{0} \ll \tau_{r} \ll T$, where $\tau_{r}$ is the characteristic time of relaxation processes $\left(\tau_{r}\right.$ is the time of relaxation to asymtotic states) and $T$ is the time of observation. The values of characteristic times in our calculations were as follows: $t_{0}=5$, $\tau_{r} \approx 60, T=2000$. At first sight, one might have expected that initial perturbations at the time interval $T$ damping at the time about $t_{0}$ would be forgotten and they will have no influence on the evolution in the large time interval. [We notice that $\tau_{r}$ is not equal to $\gamma^{-1}$, exactly $\tau_{r} \gg \gamma^{-1}$ in our case. This is connected with the system's nonlinearity. The value of 
$\tau_{r}$ is defined from numerical calculation of our problem (1), (2).] In Fig. 4 are shown the results of calculation of LI's with values of parameters for $H_{0}, L, \gamma$, and $\beta$ mentioned above and by specific values of parameter $a$. As we can see from Fig. 4 three typical clusters of states take place: a cluster of chaotic states $c h$ (in Fig. 4 the following values of the parameter $a$ correspond to them: $a=0,0.175,0.180,0.280)$, a cluster of regular states $r(a=0.290,0.300,0.320)$, and a cluster of stationary states $s(a=0.190,0.195,0.285)$. For the cluster $c h$ the values of the LI $\lambda \approx 5 \cdot 10^{-2}$; for the cluster $r, \lambda \approx-10^{-3}$; and for the cluster $s$, $\lambda \approx-10^{-1}$.

In Fig. 5 the potentials on the junction $\varphi_{t}$ depending on time for three values of parameter $a$ differing from each other on 0.005 and belonging to cluster ch, $a=0.280$ [Fig. 5a]; to cluster $s, a=0.285$ [Fig. 5b]; and to cluster $r, a=0.290$ (Fig. 5c) are showed; their LI's are represented in Fig. 4. Thus, a small change of parameter $a$ leads to a transition between all three characteristic states of the system.

In Table I transitions between chaotic ch, stationary $s$, and regular $r$ states are represented by a change of the parameter $a$ from 4.000 to 4.155 at the fixed remaining parameters indicated above.

Table I. States of a LJJ

\begin{tabular}{|c|c|c|c|c|c|c|c|}
\hline 4.000 & 4.005 & 4.010 & 4.015 & 4.020 & 4.025 & 4.030 & 4.035 \\
\hline$r$ & $r$ & $r$ & $r$ & $r$ & $r$ & $r$ & $c h$ \\
\hline
\end{tabular}

\begin{tabular}{|c|c|c|c|c|c|c|c|}
\hline 4.040 & 4.045 & 4.050 & 4.055 & 4.060 & 4.065 & 4.070 & 4.075 \\
\hline$c h$ & $c h$ & $c h$ & $s$ & $s$ & $s$ & $s$ & $s$ \\
\hline
\end{tabular}

\begin{tabular}{|c|c|c|c|c|c|c|c|}
\hline 4.080 & 4.085 & 4.090 & 4.095 & 4.100 & 4.105 & 4.110 & 4.115 \\
\hline$c h$ & $c h$ & $c h$ & $c h$ & $c h$ & $c h$ & $c h$ & $c h$ \\
\hline
\end{tabular}

\begin{tabular}{|c|c|c|c|c|c|c|c|}
\hline 4.120 & 4.125 & 4.130 & 4.135 & 4.140 & 4.145 & 4.150 & 4.155 \\
\hline$s$ & $s$ & $r$ & $r$ & $r$ & $r$ & $r$ & $r$ \\
\hline
\end{tabular}

We note that the transitions between the states, reduced in Table I and stipulated by a change of the perturbation parameter $a$, correspond to the "starting" state of chaos $(a=0)$. Thus, by the given values of 
$H_{0}, L, \gamma$, and $\beta$ a final asymptotic state is determined with the parameter $a$ independently of this, whether the "starting" state is chaotic or not. Such asymptotic behavior of the system says that dynamical chaos essentially differed from statistical chaos by which any perturbation damps rapidly and a system relaxes to its final state (for example, to the state of thermodynamical equilibrium) completely "having forgotten" an initial perturbation; i.e., the final state does not depend on this perturbation. As we see, a system in the state of dynamical chaos in contrast to a system being in the state of statistical chaos "remembers" the initial perturbation and, in any sense, final states and transitions between them are defined with the very initial perturbation. It makes it possible for us to recognize this memory effect in the system described with the sine-Gordon equation with dissipation and in the presence of an external magnetic field and a bias current. Thus, the dynamical chaos originating in a nonlinear system does not destroy an initial information; i.e., the nonlinear system has a memory in the states of dynamical chaos as well.

${ }^{1}$ W.J. Yeh, O.G. Symko, and D.J. Zheng, Phys. Rev. B 42, 4080(1990). ${ }^{2}$ L.E. Guerrero and M. Ostavio, Physica B 165-166, 1657(1990).

${ }^{3}$ L.E. Guerrero and M. Ostavio, Physica B 165-166, 1659(1990).

${ }^{4}$ M. Cirillo and N.F. Pedersen, Phys. Lett. A 90, 150(1982).

${ }^{5}$ N. Gronbech-Jensen, P.S. Lomdahl, and M.R. Samuelsen, Phys. Rev. B 43, 12799(1991).

${ }^{6}$ N. Gronbech-Jensen, Phys. Rev. B 45, 7315(1992).

${ }^{7}$ S. Rajasekar and M. Lakshmanan, Physica A 167, 793(1990).

${ }^{8}$ S. Rajasekar and M. Lakshmanan, Phys. Lett. A 147, 264(1990).

${ }^{9}$ E.F. Eriksen and J.B. Hansen, Phys. Rev. B 41, 4189(1990).

${ }^{10}$ X. Yao, J.Z. Wu, and C.S. Ting, Phys. Rev. B 42, 244(1990).

${ }^{11}$ A. Barone and G.Paterno,Physics and Applications of the Josephson Effect (Wiley-Interscience,New-York, 1982).

${ }^{12}$ K.N. Yugay, N.V. Blinov, and I.V. Shirokov, Phys. Rev. B 49, 12036 (1994).

${ }^{13}$ A.J. Lichtenberg and M.A. Lieberman, Regular and Stochastic Motion (Springer-Verlag, New-York, 1983).

${ }^{14}$ F.F. Chen, Introduction to Plasma Physics and Controlled Fusion (Plenum 
Press, New-York, 1984). 


\title{
Effect of memory and dynamical chaos in long Josephson junctions
}

\author{
K.N. Yugay, N.V. Blinov, and I.V. Shirokov
}

Fig. 1. The potential $\varphi_{t}$ (a) and the Lyapunov index (b) in a chaotic state by $\beta=0.50$. The values of other parameters are $H_{0}=1.25, L=5$, $\gamma=0.26, a=0$.

Fig. 2. The potential $\varphi_{t}$ (a) and the Lyapunov index (b) in a stationary state by $\beta=0.427$. Other parameters are the same as those in Fig. 1 .

Fig. 3. The potential $\varphi_{t}$ (a) and the Lyapunov index (b) in a regular state by $\beta=0.60$. Other parameters are the same as those in Fig. 1.

Fig. 4. The Lyapunov indices $\lambda$ vs parameter $a$ : $c h$ is the cluster of chaotic states $(a=0,0.175,0.180,0.280), r$ is the cluster of regular states ( $a=0.290,0.300,0.320)$, and $s$ is the cluster of stationary states $(a=0.190,0.195,0.285)$. The values of other parameters are just the same: $H_{0}=1.25, L=5, \gamma=0.26, \beta=0.44$.

Fig. 5. The potential $\varphi_{t}$ vs $t$ belonging to the cluster $c h, a=0.280$ (a), to the cluster $s, a=0.285$ (b), and to the cluster $r, a=0.290$ (c). Other parameters are the same as those in Fig. 4. 


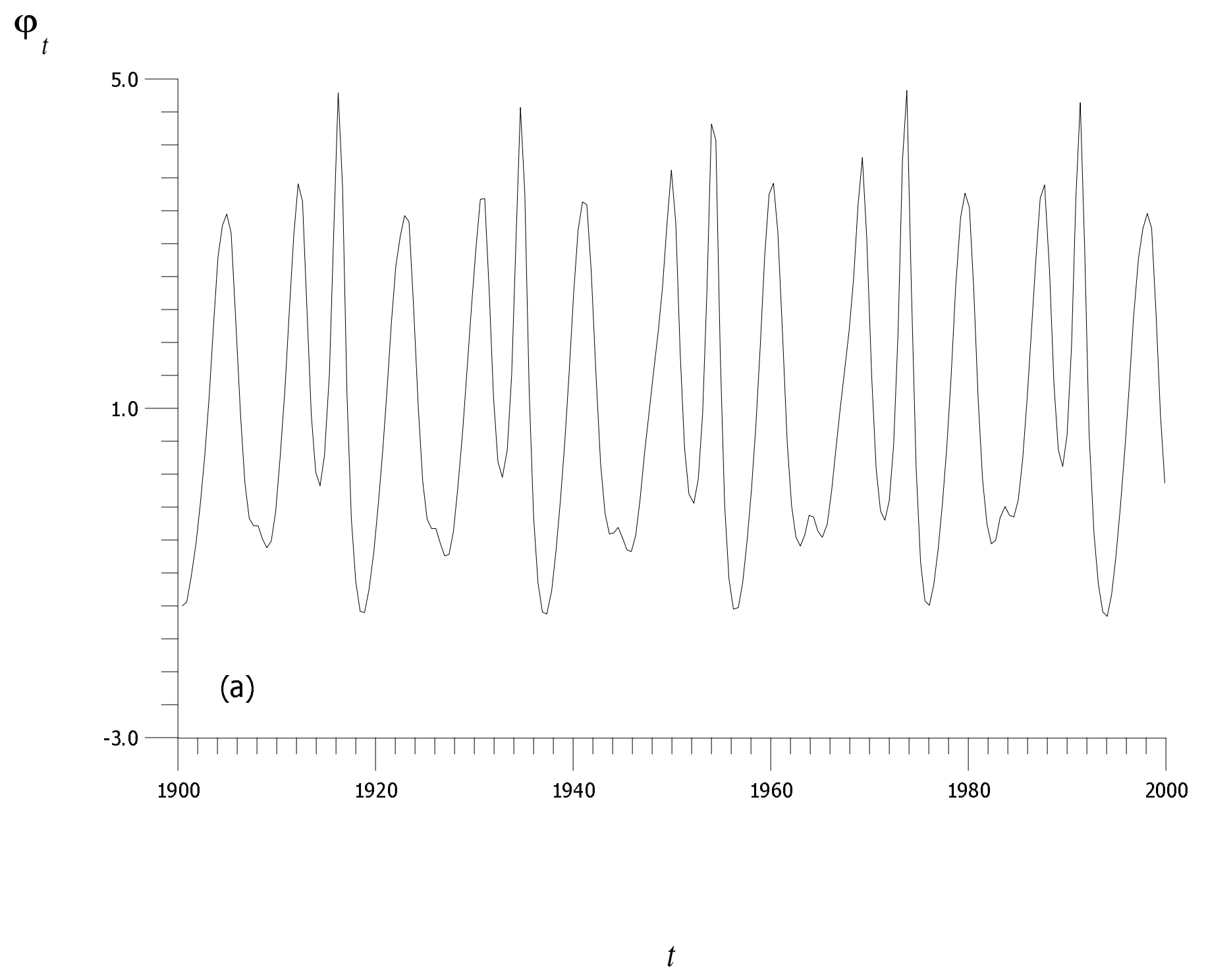




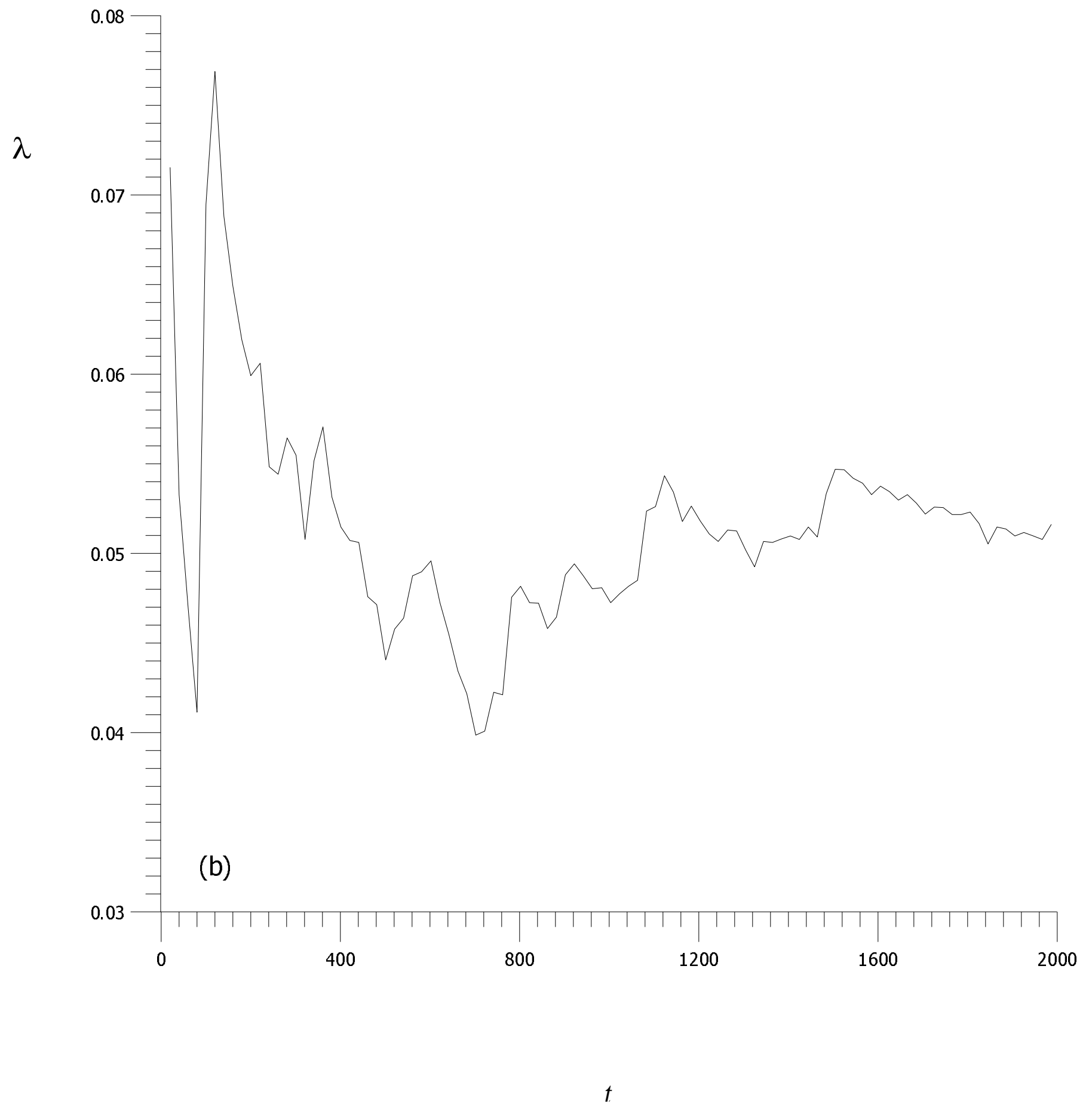




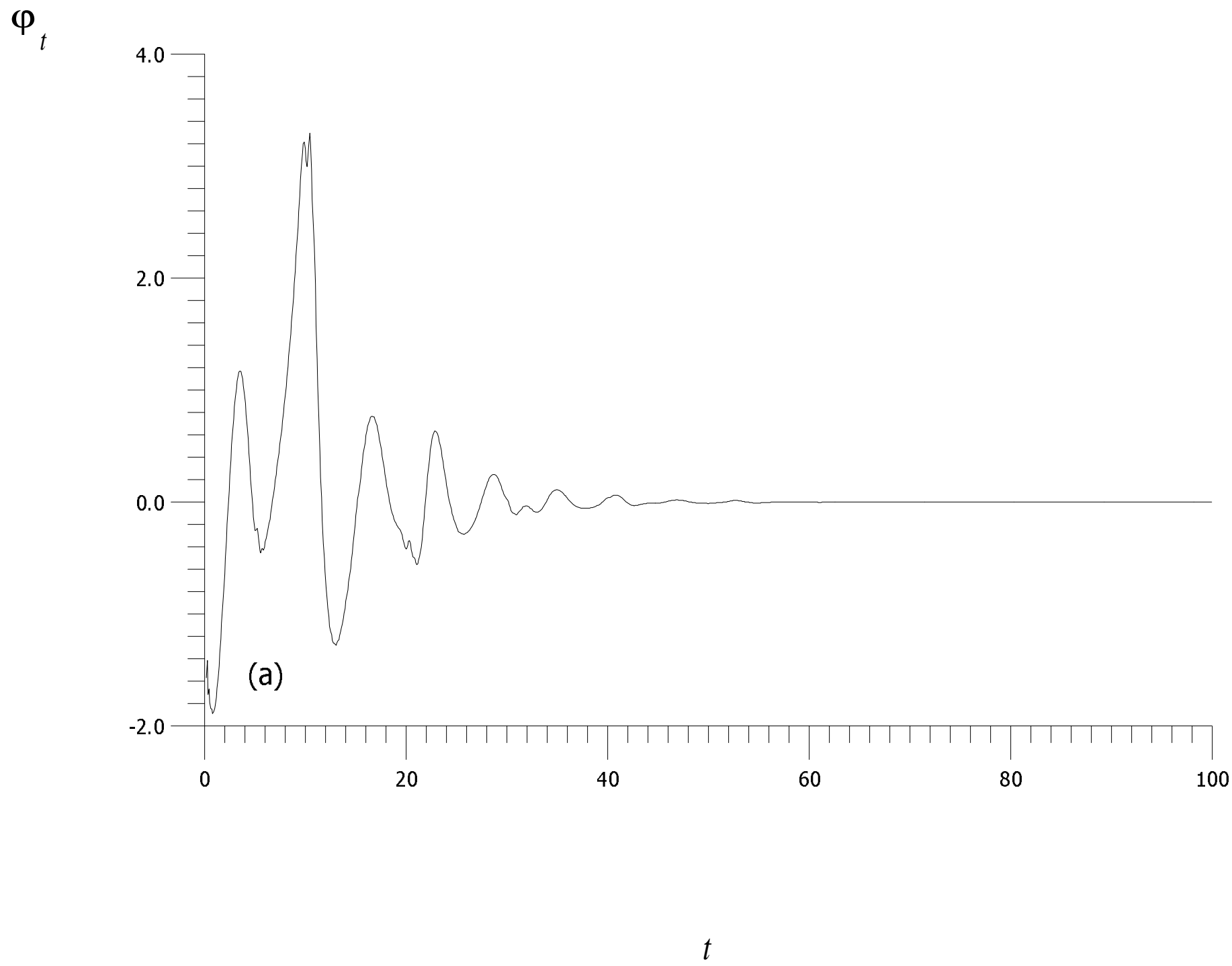




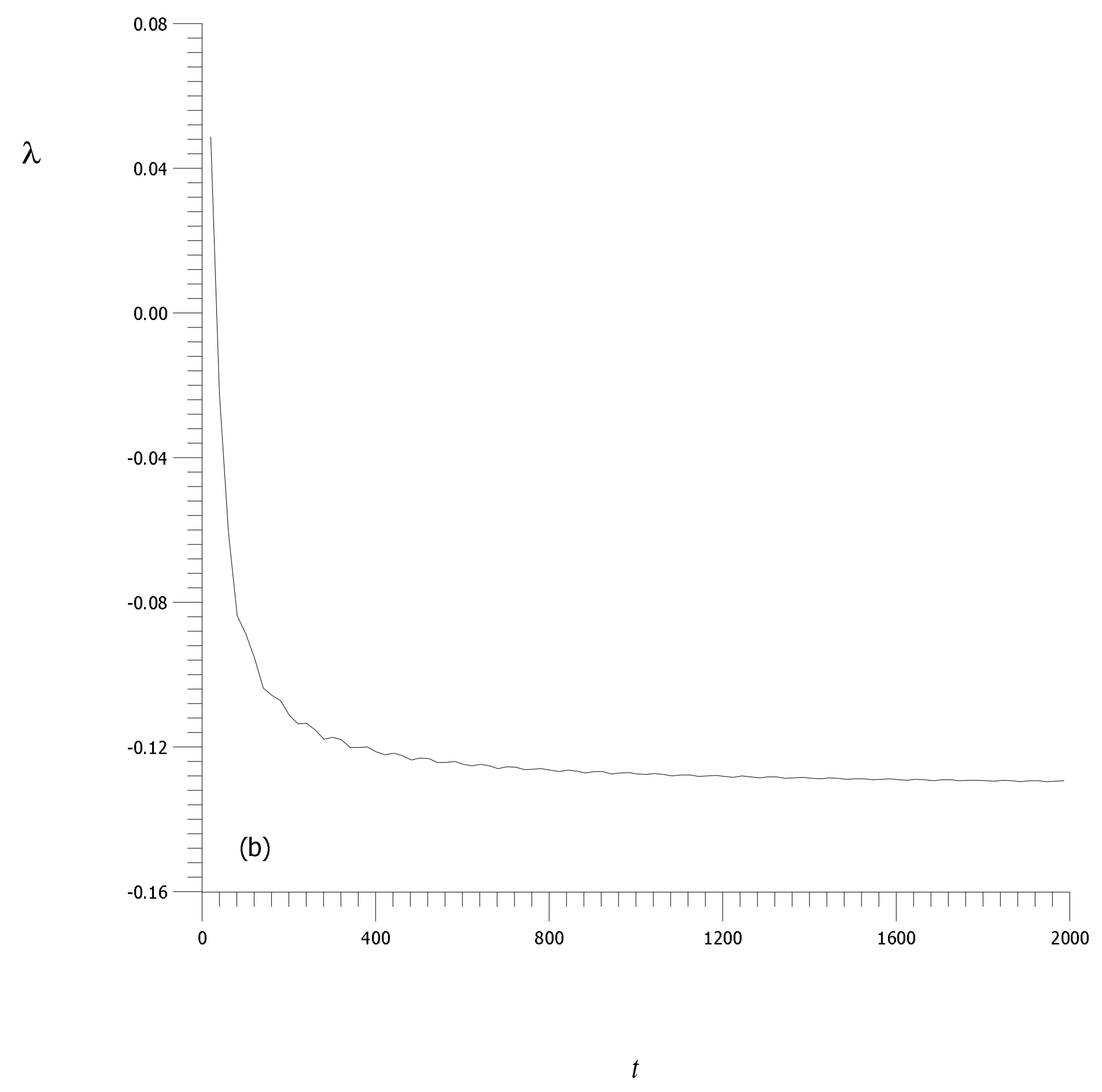




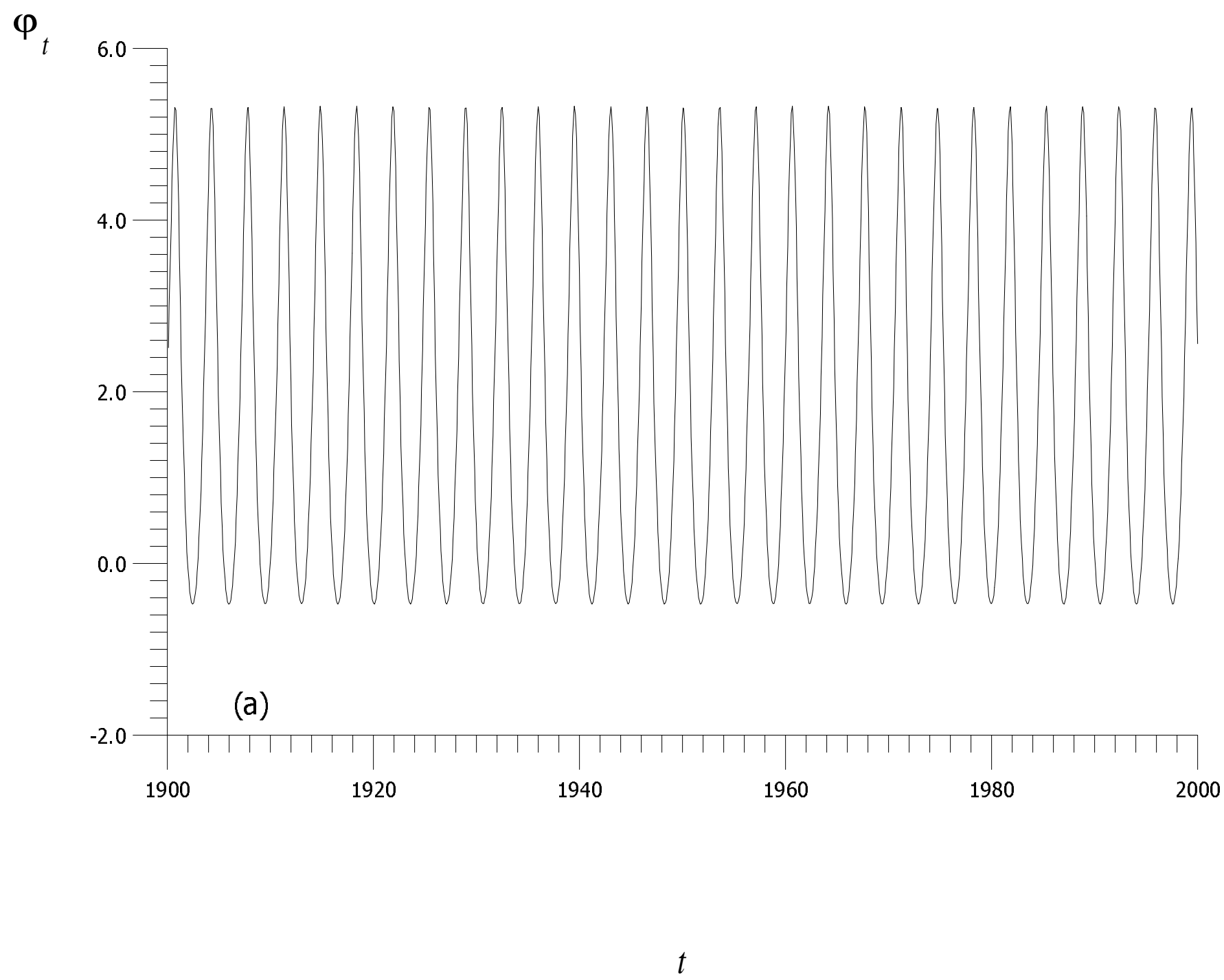




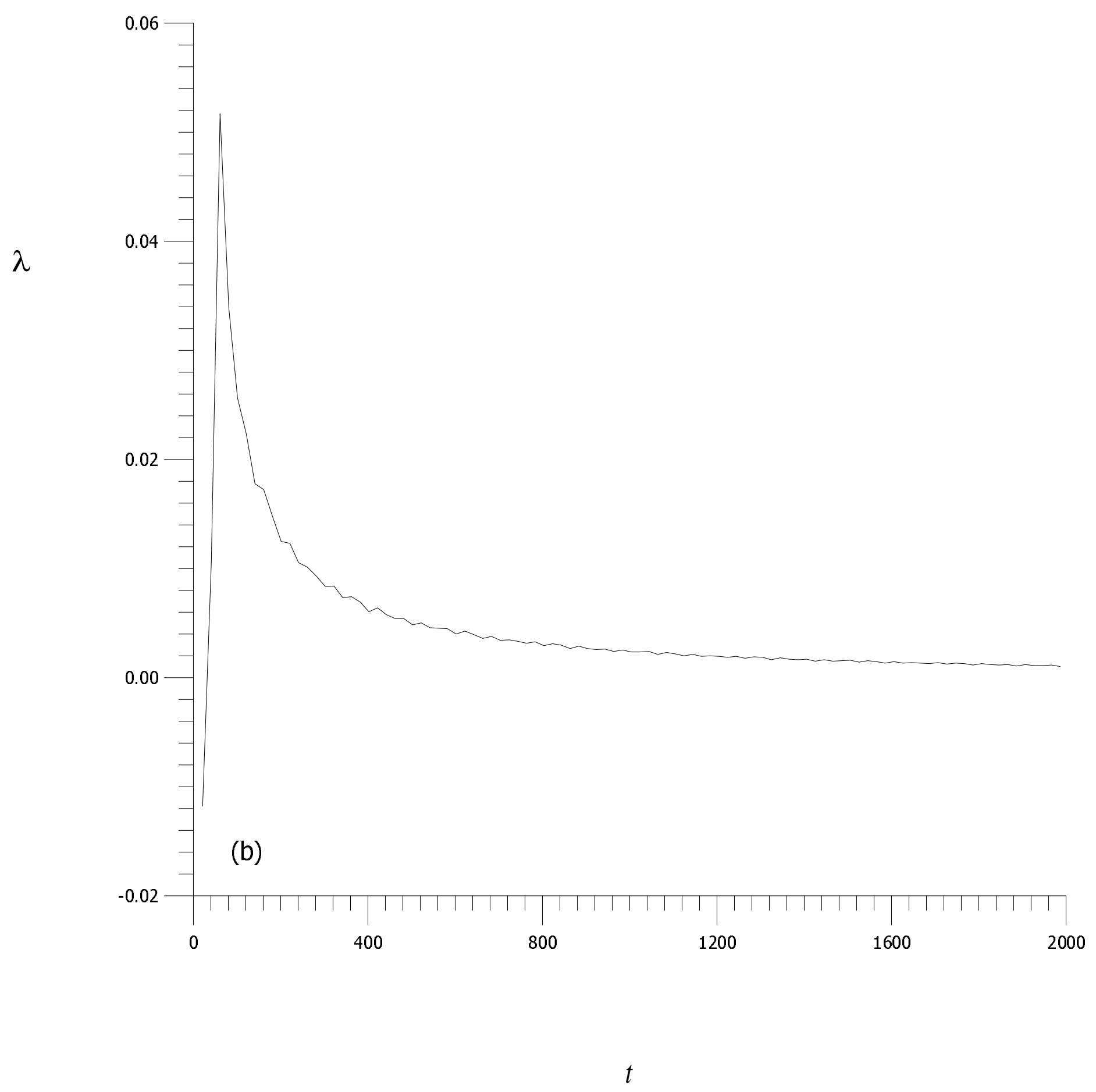




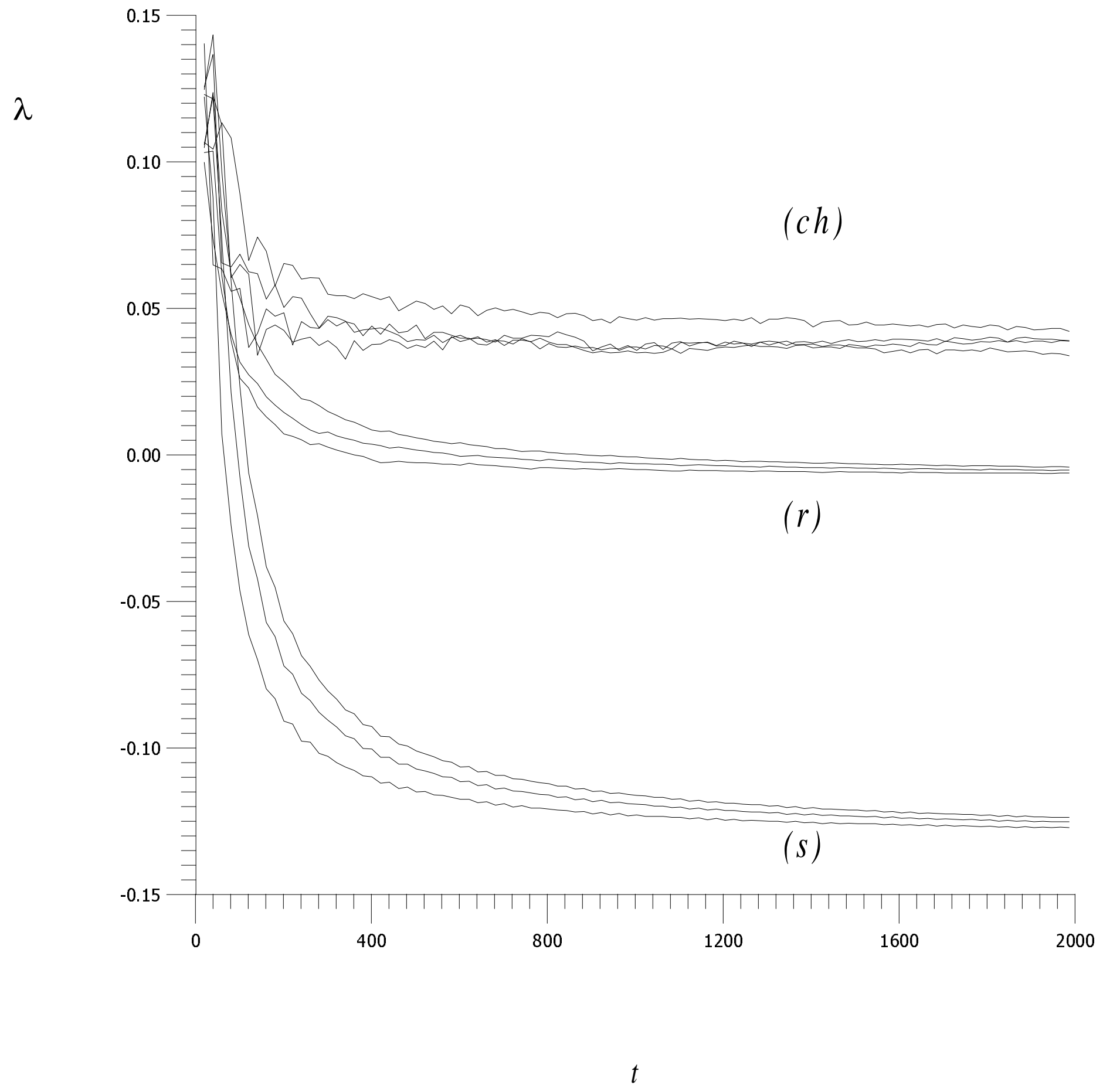




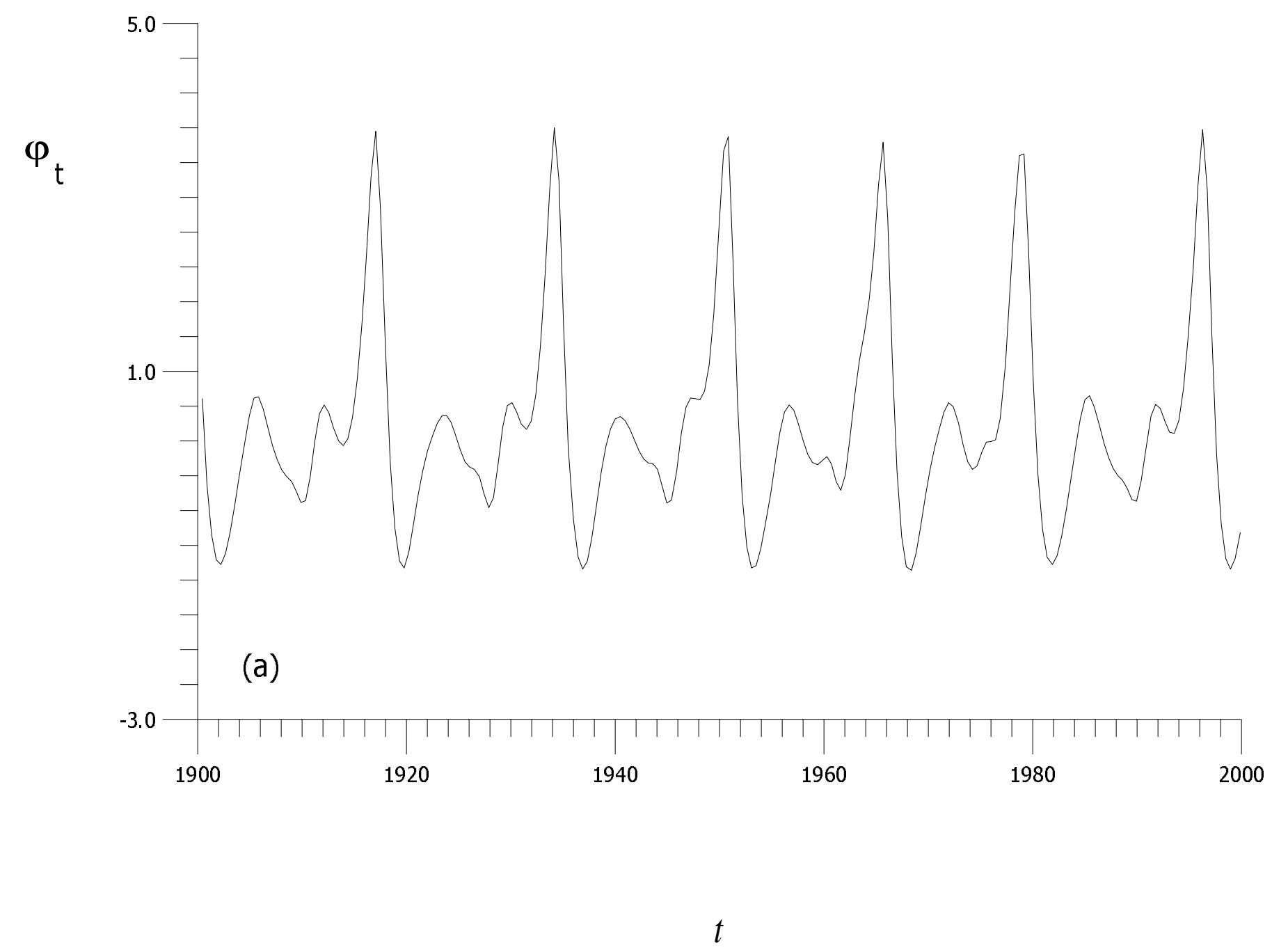




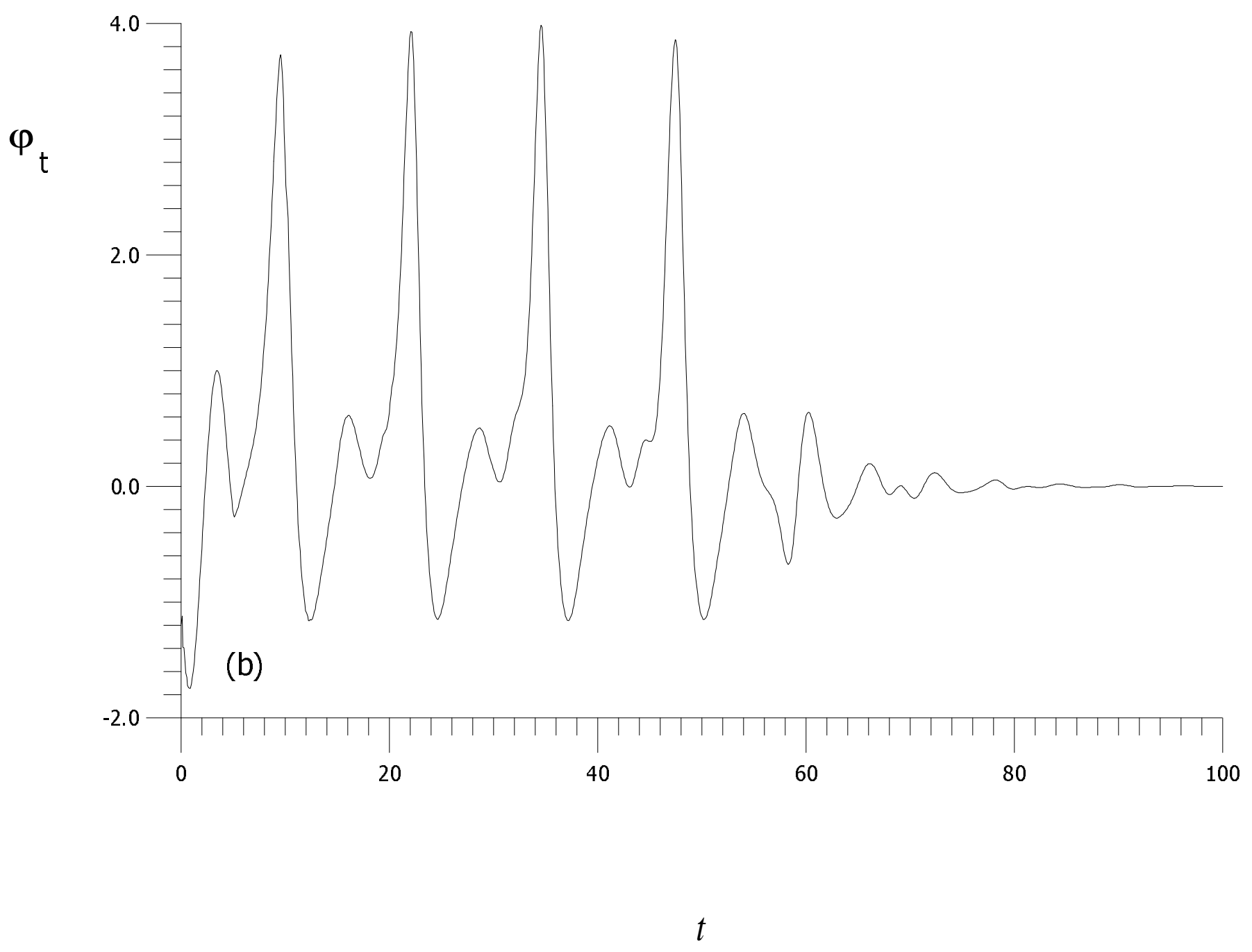




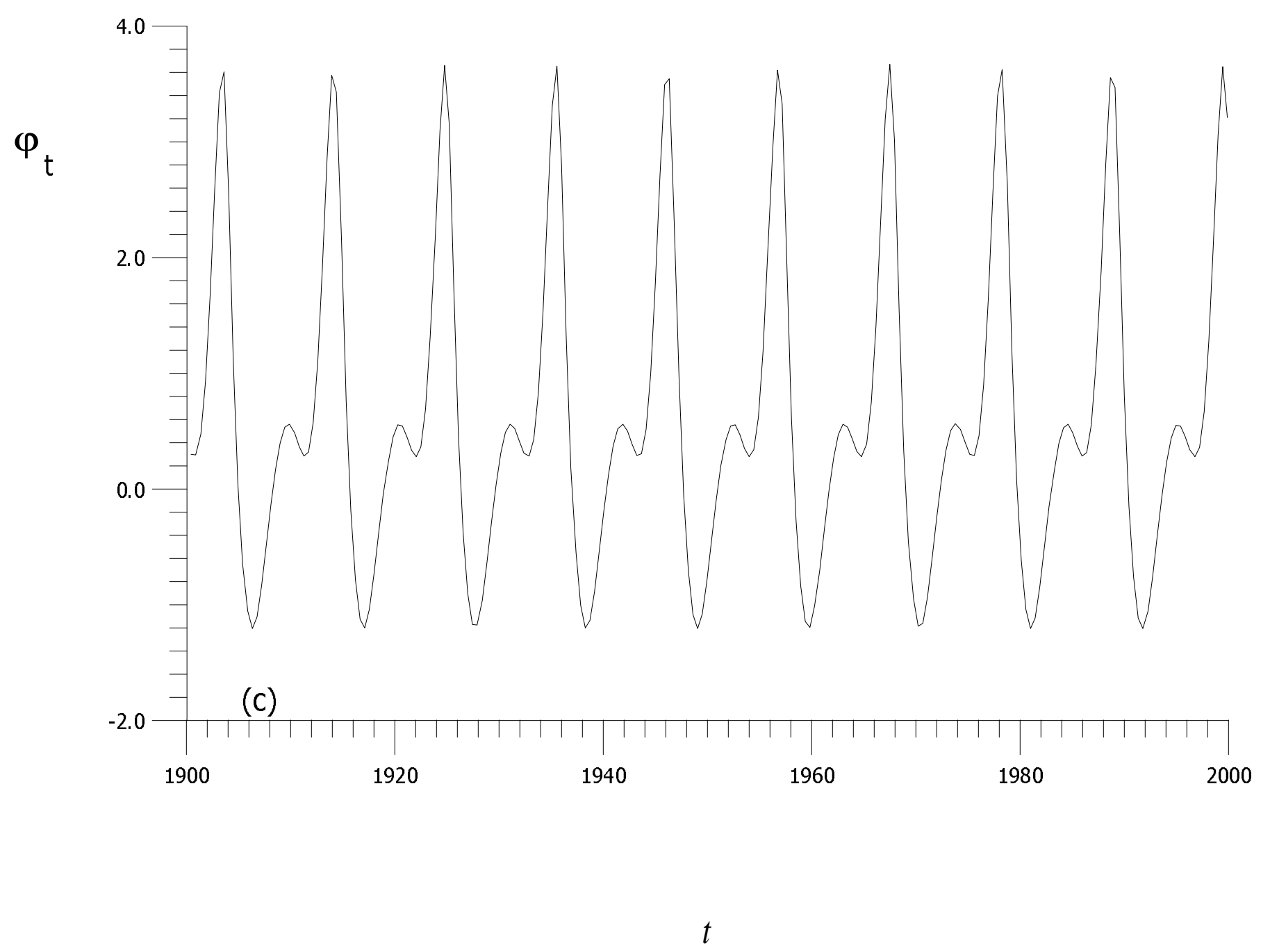

